\title{
Usefulness of sacral nerve modulation in a series of multiple sclerosis patients with bladder dysfunction
}

\author{
Elena Andretta ${ }^{\mathrm{a}}$, Claudio Simeone ${ }^{\mathrm{b}}$, Edoardo Ostardo ${ }^{\mathrm{c}}$, Mauro Pastorello ${ }^{\mathrm{d}}$, Cristina Zuliani ${ }^{\mathrm{e}, *}$ \\ a Urology Department, General Hospital of Dolo, Venice, Italy \\ b Urology Department, University of Brescia, Italy \\ c Urology Department, General Hospital “Sta. Maria degli Angeli”, Pordenone, Italy \\ d Urology Department, Sacro Cuore Hospital Negrar, Verona, Italy \\ e Neurology Department, General Hospital of Mirano, Venice, Italy
}

\section{A R T I C L E I N F O}

\section{Article history:}

Received 27 June 2014

Received in revised form 11 September 2014

Accepted 6 October 2014

Available online 12 October 2014

\section{Keywords:}

Sacral nerve modulation

Multiple sclerosis

Neurogenic bladder

Bladder storage symptoms

Bladder voiding symptoms

Bladder mixed symptoms

\begin{abstract}
A B S T R A C T
Objective: Bladder dysfunctions are common in multiple sclerosis (MS) often causing the most distressing symptoms. The aim of this paper was to evaluate the effectiveness of sacral nerve modulation (SNM) in this disease. Methods: We conducted an observational retrospective survey in 17 patients treated with SNM in the north-east of Italy, all complaining of bladder symptoms (storage in $41 \%$, voiding in $24 \%$, mixed in $35 \%$ ) unresponsive to conventional therapies, with a mean follow-up of $52 \pm 26$ months and mean Expanded Disability Status Scale score of $5.8 \pm 1.8$.

Results: $75 \%$ of patients reported significant and lasting improvement in bladder symptoms and in quality of life. We observed a statistically significant improvement in frequency, urgency, number of pads, residual volumes, number of catheterizations and in the voided volumes.

In 5 out of 6 cases with mixed symptoms the stimulation was discontinued (device totally explanted or turned off) after a mean time of 66 months (range 10-84 months) after the implant, for disease progression or loss of efficacy.

Conclusion: SNM could be an option in very selected cases of storage and voiding symptoms refractory to conservative treatments caused by a stable or slowly progressive MS considering its minimal invasiveness and reversibility. The poor results observed suggest avoiding this therapy in mixed symptoms and in cases of advanced disability.
\end{abstract}

(c) 2014 Elsevier B.V. All rights reserved.

\section{Introduction}

Multiple sclerosis (MS) is a chronic inflammatory, demyelinating, neurodegenerative disorder affecting the central nervous system (CNS) with unknown etiology [1] and it represents the commonest progressive neurological disease affecting young people.

Bladder dysfunctions are common in people with MS, occurring in up to $75 \%$ of patients during the course of the disease; they can cause some of the most distressing symptoms, with a great impact on the quality of life (QoL), and are often associated with significant morbidity [2]. Urinary symptomatology is polymorphic and, like its incidence, subject to change over time, but it tends to increase with disease progression and it is mainly the result of spinal cord involvement. The most frequent are storage symptoms (urinary frequency, urgency, and urge incontinence) while voiding symptoms (hesitancy, incomplete voiding and urinary retention) exist to a lesser degree [3]. Therapeutic options

* Corresponding author at: Neurology Department, General Hospital of Mirano, Via Mariutto, 76, 30035 Mirano, Venice, Italy. Tel.: + 39348 7812797; fax: + 390415794599

E-mail address: vdf@protec.it (C. Zuliani). for storage symptoms vary and they include, with or without associated clean intermittent self-catheterization, conservative treatments such as anticholinergic drugs - although the published data provide limited evidence for their efficacy in MS [4,5]. In patients with severe bladder overactivity unresponsive to anticholinergics there is evidence for the use of bladder injections of botulinum toxin $A$ [6,7] and also emerging evidence on the use of cannabinoids [8] and tibial nerve stimulation [9]. Surgical procedures, which have been advocated with varying success [10], are a last resort being irreversible, with a risk of major operative morbidity and long-term consequences; they include augmentation cystoplasty [11], in which the bladder is transected and repaired with a patch of ileum isolated from the small intestine, and urinary diversion [12].

Sacral nerve modulation (SNM) is a safe and minimally invasive treatment and it has become an option in refractory low urinary tract symptoms (LUTS) from different underlying diseases [13-16]; a number of prospective trials and numerous case series have provided an evidence base that has confirmed the efficacy and durability of SNM [17]. Despite the documented clinical benefit, the exact underlying neurophysiological mechanism of action of SNM is complex and not fully 
understood. However, evidence supports an emerging consensus that it exerts its effect through afferent pathways that modulate the activity in other neural pathways within the spinal cord and higher centers [18]. Some studies have just investigated the effects of SNM on the higher centers. Braun et al. [19] performed serial electroencephalograms (EEG) in 10 patients with both neurogenic and idiopathic bladder dysfunction who had permanent implants of SNM in S3, using an on-off paradigm with $1.5 \mathrm{~s}$ with stimulation "on" followed by a 10 second stimulation "off". They demonstrated in all patients a cortical potential complex following stimulation with an early electronegative component at 50 ms followed by a late potential component with a mean latency of $253 \mathrm{~ms}$, both with a maximum in the post-central gyrus. These findings occurred irrespective of patients' reports of actually feeling the SNM being switched on and off, whereby the authors concluded that, although these potentials are similar to cognitively mediated "event-related potentials", they are clearly distinct from any subjective sensory or even painful response and support the potential role of suprasacral centers in SMN, most probably in the sensory cortex areas [19].

Blok et al. [20] used positron emission tomography (PET) to study the brain effects of SNM in patients with urge incontinence comparing chronic (in whom SNM was switched on for $>6$ months) and acute patients (neurostimulator activated for the first time in PET scanner). They observed different modifications in cerebral blood flow in different areas during the chronic and acute stimulations. On the basis of these findings, the authors suggested that chronic SNM influences, presumably via the spinal cord, brain areas previously implicated in bladder overactivity, awareness of bladder filling, the urge to void and the timing of micturition and, furthermore, areas involved in alertness and awareness. On the other hand acute SNM modulates predominantly areas involved in sensorimotor learning, which might become less active during the course of chronic SNM [20].

Since few data are available about this treatment for LUTS in MS [21-23], in the present retrospective evaluation we wanted to determine whether SNM benefits patients with LUTS caused by this disease and unresponsive to first line conservative treatment and to assess if there is any difference in the responsiveness degree of different symptoms.

\section{Materials and methods}

In July 2008, we proposed to the Departments of Urology in the north-east of Italy performing SNM a retrospective survey about the effectiveness of SNM in MS patients. Only in 3 departments was SMS used in MS patients and all the MS patients implanted in these centers were included in the survey. A questionnaire has been filled for each included subject, enquiring about general data, MS features, perceived changes in micturition symptoms after SNM and changes coming from micturitiondiaries. The post-voiding residual volume was evaluated by the clean intermittent catheterization which is usually performed by patients with voiding and mixed symptoms.

A visual analogic scale (VAS) was used to assess the improvement of the QoL and the symptom urinary urgency. Outcome data were obtained from the periodic follow-up as usually conducted.

For statistical analysis the following tests were applied: Cramer's V test, Kruskal Wallis test, univariate ANOVA, true Fisher test.

The clinical practice followed with uniformity by all the Departments required that the candidates for surgical implantation were selected on the basis of informed consent, prolonged failure of the conservative treatments with severe impact on QoL, no abnormality of upper urinary tract and kidney function. The pre-operative assessment included a 5 day voiding diary, the post-void residual evaluation, serum creatinine determination, urine culture, kidney ultrasound and videourodynamics. In the voiding diaries were recorded also the leakage episodes, degree of urgency and the pad used. The SNM implant procedure was performed as previously described [24].
The test period lasted for at least 3 weeks and the stimulation parameters (pulse width, frequency and amplitude) were individually chosen for each patient on the basis of the best responses, with stimulation below threshold. Eligibility criteria for the definitive implant were represented by a positive neuroanatomical response to the stimulation with clinical improvement in the symptoms and no side effects.

\section{Results}

17 subjects agreed to complete the survey during a follow-up visit, 13 females and 4 males, mean age $49.8 \pm 10.9$ years. The disease duration before implant ranged from 4 to 40 years (mean $13.5 \pm 9.2$ years); 10 subjects (59\%) had the relapsing remitting, 2 (12\%) the secondary progressive and 5 (29\%) the primary progressive forms of the disease, with a mean Expanded Disability Status Scale (EDSS) score of $5.8 \pm$ 1.8 [25]. Thirteen subjects (77\%) were paraparetic. Regarding LUTS, 4 patients (24\%) had voiding symptoms, 7 (41\%) storage symptoms and 6 (35\%) mixed symptoms; 6 patients reported simultaneous constipation. No statistical differences were identified among these three groups in baseline characteristics except for the anal tone, which resulted hypertonic in $83 \%$ of patients with mixed symptoms $(p=0.032)$. The mean baseline EDSS score was worse in mixed and voiding LUTS than in the storage ones. The mean time from the implant was $52 \pm$ 26 months (range 12-97 months). The electrical parameters have been individually programmed and the number of reprogramming during the period of observation ranged from an average of 0.9 , in patients with storage symptoms, to 3.0, in those with mixed LUTS. In five out of 6 cases with mixed symptoms the stimulation has been discontinued after a mean time of 66 months (range 10-84 months) for disease progression in 2 cases and for loss of efficacy in 3. The mean EDSS score didn't change significantly from baseline to the last follow-up evaluation, but patients complaining of mixed symptoms presented a quicker progressive MS (Table 1).

Regarding the subjective improvements, $75 \%$ of the total population ( $100 \%$ of patients with storage, $75 \%$ with voiding and $40 \%$ with mixed symptoms) replied positively to the question "After SNM did you detect any significant and lasting improvement in your bladder symptoms?"

The change in QoL was evaluated by the VAS after the question "How much has your QoL changed?" with a score ranging from 0 (not at all) to 5 (very much). The average score reported was $2.9 \pm 1.5$ for the whole population, $1.8 \pm 1.4,3.3 \pm 1.0$ and $3.6 \pm 1.4$ for patients respectively with mixed, storage and voiding symptoms (Fig. 1). Furthermore, there was a statistically significant reduction in subjective urgency level evaluated by the VAS - ranging from 0 (no urgency at all) to 5 (severe urgency) - in patients with storage and mixed symptoms; they passed from an average score of $3.6 \pm 1.1$ before SNM to $2.0 \pm 1.3$ during SNM ( $\mathrm{p}=0.014$ ) (Fig. 2).

Analyzing the subgroups, there was a statistically significant reduction in the number of micturitions/day (from $14.3 \pm 9.6$ to $8.1 \pm 3.7$; $\mathrm{p}=0.012$ ) and number of pads/day (from $3.5 \pm 1.8$ to $1.9 \pm 2.4$; $\mathrm{p}=0.033$ ) in patients with storage and mixed symptoms. In subjects with voiding and mixed symptoms was found a statistically significant reduction in number of catheterizations/day (from $3.1 \pm 2.0$ to $0.9 \pm$ $1.0 ; \mathrm{p}=0.017$ ) and residual volumes (from $195.0 \pm 9.0$ to $71.5 \pm$ $63.3 \mathrm{ml} ; \mathrm{p}=0.018$ ) and a statistically significant increase in

Table 1

EDSS score in different LUTS, at baseline and during SNM.

\begin{tabular}{lrll}
\hline & N & Baseline EDSS & Follow-up EDSS \\
\hline All patients, mean (SD) & 17 & $5.8(1.8)$ & $6.3(2.1)$ \\
Voiding symptom, mean (SD) & 4 & $6.5(1.8)$ & $6.5(1.9)$ \\
Storage symptoms, mean (SD) & 7 & $5.1(2.1)$ & $5.4(2.2)$ \\
Mixed symptoms, mean (SD) & 6 & $6.8(0.5)$ & $7.9(0.9)$ \\
\hline
\end{tabular}




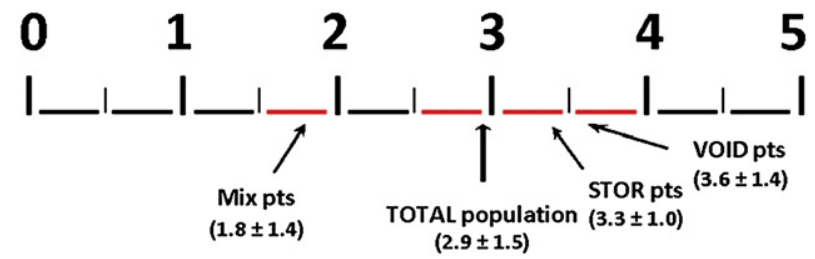

Fig. 1. Quality of life changes with SNM at the time of survey compilation. Score ranging from 0 (not at all) to 5 (very much).

spontaneous voided volumes (from $131.9 \pm 36.7$ to $209 \pm 90.0 \mathrm{ml}$; $\mathrm{p}=0.016$ ). In Figs. 3, 4, 5, 6 and 7 are shown these changes.

\section{Discussion}

Schmidt et al. introduced SNM for the treatment of different kinds of LUTS refractory to conservative treatments [26]. In 2000 a multicenter, international and prospective study of 581 patients found that SNM reduced by more than $50 \%$ the voiding frequency, incontinence episodes and retention symptoms in patients with such intractable conditions, but the study excluded patients with underlying neurologic disorders [27].

SNM has been also used in some cases of neurogenic bladder [14,16, 28 ] and also in neurogenic bladder caused by MS, though the few references usually regard a small number of patients and mainly with a short or median follow-up [21-23,29]. Furthermore the use of SNM is discouraged in MS for the progressive features of the disease [4] and a limitation to the use of this therapy in MS patients is its contraindication to magnetic resonance (MRI) examination.

In many MS patients the demyelination of the dorsal columns, which determines the interruption of normal inhibitory signals coming from sacral parasympathetic outflow tracts, can result in neurogenic detrusor overactivity, which represents the most common problem experienced during the course of the disease. Also voiding symptoms can be experienced by MS patients when the urethral sphincter fails to relax in a picture of detrusor-sphincter dyssynergia [3] or in case of detrusor hypocontractility, reported in $20 \%$ of cases [30].

Another problem is the fact that MS nervous system lesions tend to resolve and then recur in different patterns, resulting in unpredictable changes in symptoms over time, and in any case this disease is generally progressive, and hence the therapeutic response can vary. Therefore patients have to be clearly informed that the results of SNM may be altered by the evolution of their underlying disease.

Notwithstanding these considerations, many authors do not consider MS a contra-indication for SNM. The few studies published on this topic regard mainly urinary incontinence; only Marinkovic and Gillen investigated pure urinary retention, reporting remarkably good results [22].

Bosch and Groen observed in 4 MS women with refractory urgeincontinence a reduction in incontinence episodes - from a mean of 4 to 0.3 per day - with SNM. Two women were completely dry and $1 \mathrm{im}-$ proved significantly during the two year follow-up; in the last case the urinary symptoms worsened with clinical progression of the disease [21]. Subsequently their 4 cases were included in a series of 9 patients [14] with neurogenic urge-incontinence resistant to conservative therapies, due to MS in overall 5 cases. At 6 months, SNM resulted in all of

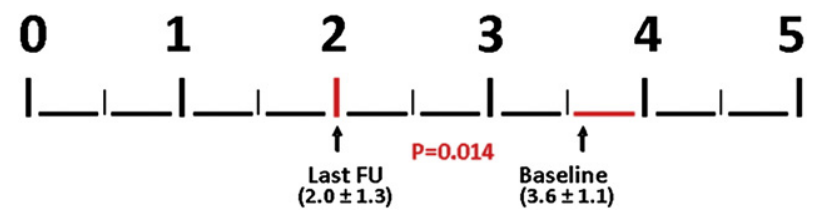

Fig. 2. Subjective urgency level at baseline (retrospective) and at the time of survey compilation. Score ranging from 0 (no urgency at all) to 5 (severe urgency).

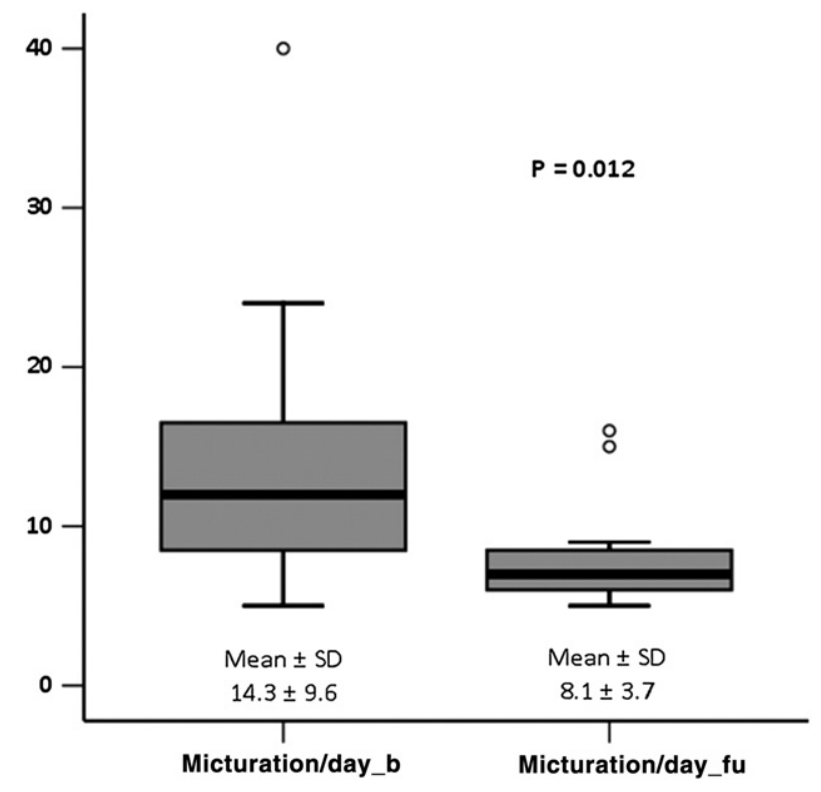

Fig. 3. Micturition number/day at baseline (b) and last follow-up visit (fu).*Strong outlier values; 'outlier value.

them having a marked symptomatic improvement in incontinence with positive changes in everyday life: frequency improved from 16.1 to 8.2 voids per day and the mean volume per void correspondingly increased from 115 to $249 \mathrm{ml}$ with six patients completely dry. These results remained stable for an average of 43.6 months except that in two MS patients. Moreover another MS patient had a short follow-up (11 months). In conclusion 2 out of 5 subjects with MS have had a long-lasting benefit from SNM with a follow-up of 60 and 72 months.

Wallace et al. implanted 28 patients with neurogenic bladder complaining of incontinence and/or urinary retention and among them there were 13 MS. At a mean follow-up of 12.4 months they observed a statistically significant improvement in all the usual items regarding detrusor overactivity and also a reduction of $58 \%$ in the number of self-catheterizations. Unfortunately MS patients are not distinguishable from other patients [16].

Recently Marinkovic and Gillen reported complete success in $86 \%$ of 14 MS females complaining of urinary retention: the pre-operative

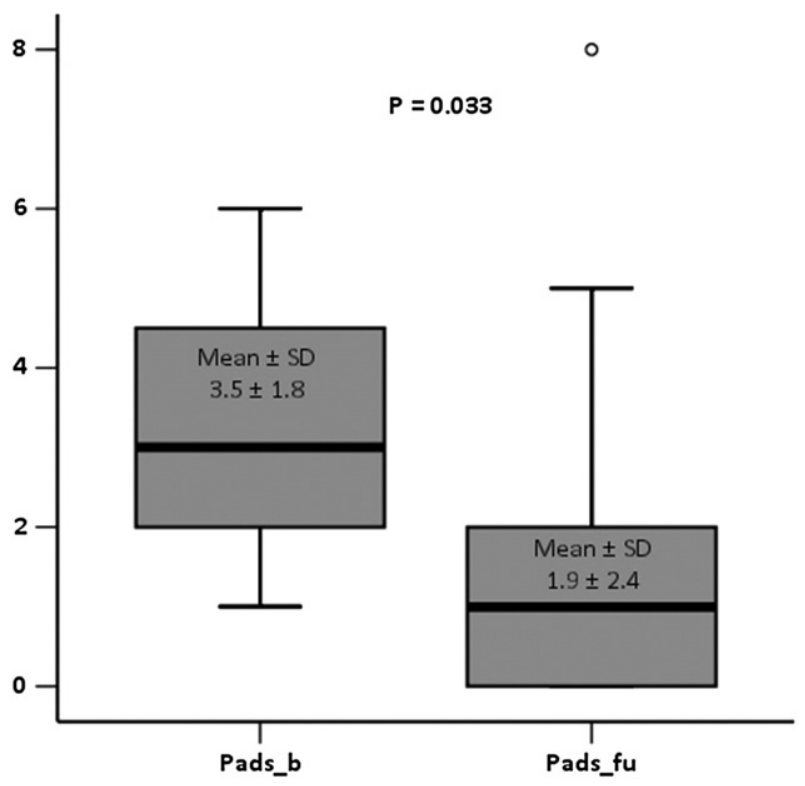

Fig. 4. Number of pads/day at baseline (b) and last follow-up visit (fu). ${ }^{\circ}$ Outlier value. 


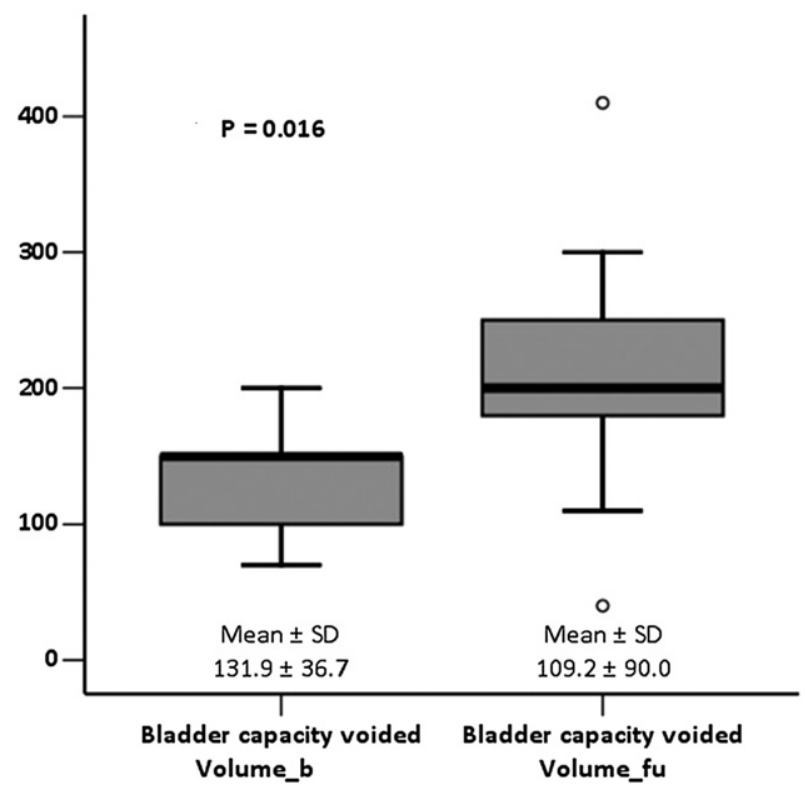

Fig. 5. Voided volume (ml) at baseline (b) and last follow-up (fu). ${ }^{\circ}$ Outlier value.

mean post-void residual of $308 \mathrm{ml}$ fell to $50 \mathrm{ml}$ and 12 women went on to void without intermittent catheterization at a follow-up of 4.3 years. The authors outlined that the only 2 patient non-responders to SNM were wheelchair-bound [22].

Minardi and Muzzonigro treated 15 patients and found that urinary retention due to detrusor underactivity is not a good indication for SNM while it should be offered in the case of refractory urge-incontinence or urinary retention due to detrusor sphincter dyssynergia [23].

Most of these studies are included in a meta-analysis about neurogenic bladder dysfunction treated with SNM which confirms the low number of MS patients investigated and their heterogeneity, with 50 MS cases out of 357 patients [29].

In none of the above studies reported have there been significant complications.

Also if the literature concerning SNM in MS is small, SNM seems to have an inhibitory effect on neurological detrusor overactivity also if the proportion of patients whose LUTS improved is much less than in non-neurological pathologies.

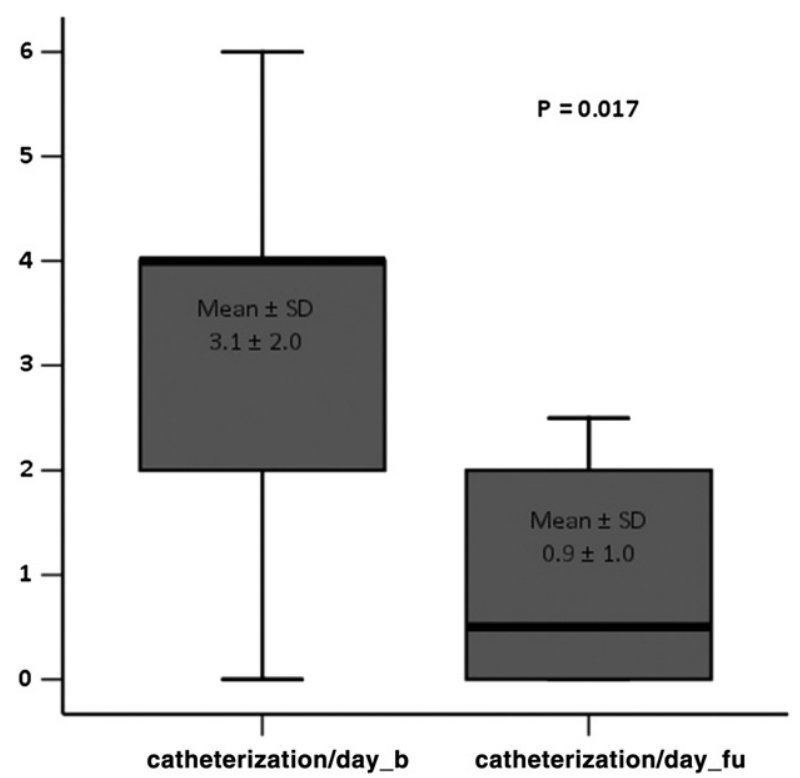

Fig. 6. Catheterization number/day at baseline (b) and last follow-up visit (fu).

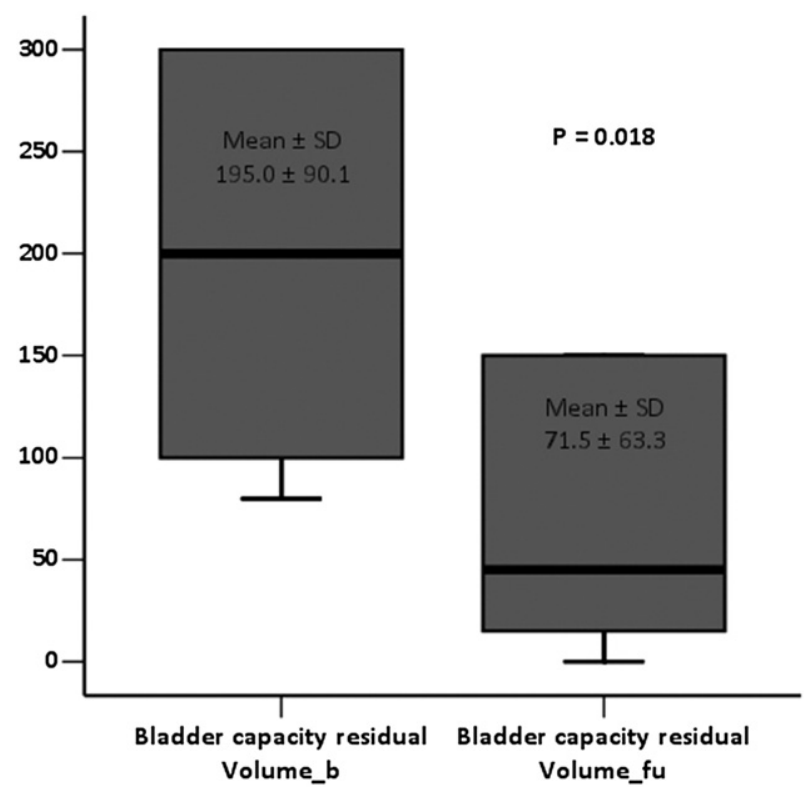

Fig. 7. Residual volume (ml) at baseline (b) and last follow-up visit (fu).

Our observational retrospective survey, regarding patients treated with SNM for every type of LUTS, is the largest MS series studied to date. SNM has been performed in a minority of Urology Units - some of those more trained in neuro-urology - and overall has been rarely used considering that only 17 patients had been implanted in a wide geographic area.

In our experience, SNM has been a quite effective therapy for LUTS with improvement in QoL in MS with lower EDSS. In fact, SNM has worked better in patients with less severe motor impairment as reported in previous studies $[22,29]$. In the present report the lack of a validated questionnaire on QoL could be a limitation but a VAS evaluation was easier to perform, equally reliable and supported by objective urinary data (reduction of leakage episodes and residual volume).

Moreover these results have been maintained over time in most of patients and they have been detected in all the items at a medium follow-up. The best responses have been observed in storage LUTS, followed by the voiding ones. Poor and short-lasting benefits have been reported in the mixed LUTS, in connection with a quicker and more aggressive form of the disease (EDSS score worsened in the course of time compared to the other cases), and this could explain the decrease of SNM efficacy over time observed in the majority of these patients (five out of six patients).

There is a significant feature in our series of patients: it is not a selected cohort but represents the real MS population. Regarding the gender, our casuistry - with 4 men out of 17 patients - reflects the gender prevalence ratio of MS, while in others reports are mainly analyzed with only female patients. Furthermore we have analyzed every type of LUTS whereas others authors focused only on incontinence or retention $[12,21-23,29]$.

\section{Conclusion}

In our experience SNM has obtained objective and subjective improvement of LUTS in most of the cases of MS with long-lasting benefit. Therefore it could be a therapeutic option in very selected cases of storage and voiding symptoms, refractory to conservative treatments, caused by a stable or slowly progressive MS without severe motor impairment.

Storage LUTS have the best chance of amelioration, followed by the voiding ones. SNM should be avoided in mixed symptoms and in cases 
of advanced disability, in order to maximize the cost-benefit of this therapy.

\section{References}

[1] Noseworthy JH, Lucchinetti C, Rodriguez M, Weinshenker BG. Multiple sclerosis. N Engl J Med 2000;343:938-52.

[2] Marrie RA, Cutter G, Tyry T, Vollmer T, Campagnolo D. Disparities in the management of multiple sclerosis-related bladder symptoms. Neurology 2007;68:1971-8.

[3] Betts CD, D'Mellow MT, Fowler CJ. Urinary symptoms and the neurological features of bladder dysfunction in multiple sclerosis. J Neurol Neurosurg Psychiatry 1993;56: 245-50.

[4] Fowler CJ, Panicker JN, Drake M, Harris C, Harrison SCW, Kirby M, et al. A UK consensus on the management of the bladder in multiple sclerosis. J Neurol Neurosurg Psychiatry 2009;80:470-7.

[5] Nicholas R, Young C, Friede T. Bladder symptoms in multiple sclerosis: a review of pathophysiology and management. Expert Opin Drug Saf 2010;9:905-15.

[6] Karsenty G, Denys P, Amarenco G, De Seze M, Gamé X, Haab F, et al. Botulinum toxin A (Botox) intradetrusor injections in adults with neurogenic detrusor overactive/ neurogenic overactive bladder: a systematic literature review. Eur Urol 2008;53: 275-87.

[7] Schulte-Bauklok H, Schobert J, Stolze T, Stuzebecher B, Weiss C, Knispel HH. Efficacy of botulinum-A toxin bladder injections for the treatment of neurogenic detrusor overactivity in multiple sclerosis patients: an objective and subjective analysis. Neurourol Urodyn 2006;25:110-5.

[8] Freeman RM, Adekanmi O, Waterfield MR, Waterfield AE, Wright D, Zajicek J. The effect of cannabis on urge incontinence in patients with multiple sclerosis: multicentre, randomised placebo-controlled trial (CAMS-LUTS). Int Urogynecol J Pelvic Floor Dysfunct 2006;17:636-41.

[9] de Sèze M, Raibault P, Gallien P, Even-Schneider A, Denys P, Bonniaud V, et al. Transcutaneous posterior tibial nerve stimulation for treatment of the overactive bladder syndrome in multiple sclerosis: results of a multicenter prospective study. Neurourol Urodyn 2011;30:306-11.

[10] Foon R. The overactive bladder. Ther Adv Urol 2010;2:147-55.

[11] Chapple CR, Bryan NP. Surgery for detrusor overactivity. World J Urol 1998;16: 268-73.

[12] Yong SM, Dublin N, Pickard R, Cody DJ, Neal DE, N'Dow J. Urinary diversion and bladder reconstruction/replacement using intestinal segments for intractable incontinence or following cystectomy. Cochrane Database Syst Rev 2003;1:CD003306.

[13] Schmidt RA, Jonas U, Oleson KA, Janknegt RA, Hassouna MM, Siegel SW, et al. Sacral nerve stimulation for treatment of refractory urinary urge incontinence. Sacral Nerve Stimulation Study Group. J Urol 1999;16:352-7.

[14] Chartier-Kastler EJ, Ruud Bosch JL, Perrigot M, Chancellor MB, Richard F, Denys P. Long-term results of sacral nerve stimulation (S3) for the treatment of neurogenic refractory urge incontinence related to detrusor hyperreflexia. J Urol 2000;164: 1476-80.

[15] Janknegt RA, Hassouna MM, Siegel SW, Schmidt RA, Gajewski JB, Rivas DA, et al. Long-term effectiveness of sacral nerve stimulation for refractory urge incontinence. Eur Urol 2001;39:101-6.

[16] Wallace PA, Lane FL, Noblett KL. Sacral nerve neuromodulation in patients with underlying neurologic disease. Am J Obstet Gynecol 2007;197:96 e. 1-5.

[17] Brazzelli M, Murray A, Fraser C. Efficacy and safety of sacral nerve stimulation for urinary urge incontinence: a systematic review. J Urol 2006;175:835-41.

[18] Craggs M, McFarlane J. Neuromodulation of the lower urinary tract. Exp Physiol 1999;84:149-60.

[19] Braun PM, Baezner H, Seif C, Boehler G, Bross S, Eschenfelder CC, et al. Alterations of cortical electrical activity in patients with sacral neuromodulator. Eur Urol 2002;41: 562-6.

[20] Blok BF, Groen J, Bosch JL, Veltman DJ, Lammertsma AA. Different brain effects during chronic and acute sacral neuromodulation in urge incontinent patients with implanted neurostimulators. BJU Int 2006;98:1238-43.

[21] Bosch JLHR, Groen J. Treatment of refractory urge urinary incontinence with sacral spinal nerve stimulation in multiple sclerosis patients. Lancet 1996;348:717-9.

[22] Marinkovic SP, Gillen LM. Sacral neuromodulation for multiple sclerosis patients with urinary retention and clean intermittent catheterization. Int Urogynecol J 2010;21:223-8.

[23] Minardi D, Muzzonigro G. Sacral neuromodulation in patients with multiple sclerosis. World J Urol 2012;30:123-8.

[24] Spinelli M, Giardiello G, Gerber M, Arduini A, van den Hombergh U, Malaguti S. New sacral neuromodulation lead for percutaneous implantation using local anesthesia: description and first experience. J Urol 2003;170(5):1905-7.

[25] Kurtzke JF. Rating neurologic impairment in multiple sclerosis: an expanded disability status scale (EDSS). Neurology 1983;33:1444-52.

[26] Schmidt RA, Senn E, Tanagho EA. Functional evaluation of sacral nerve root integrity. Report of a technique. Urology 1990;35:388-92.

[27] Siegel SW, Catanzaro F, Dijkema HE, Elhilali MM, Fowler CJ, Gajewski JB, et al. Longterm results of a multicenter study on sacral nerve stimulation for treatment of urinary urge incontinence, urgency-frequency, and retention. Urology 2000;56:87-91.

[28] Chaabane W, Guillotreau J, Castel-Lacanel E, Abu-Anz S, De Boissezon X, Malavaud B, et al. Sacral neuromodulation for treating neurogenic bladder dysfunction: clinical and urodynamic study. Neurourol Urodyn 2011;30:547-50.

[29] Kessler TM, La Framboise D, Trelle S, Fowler CJ, Kiss G, Pannek J, et al. Sacral neuromodulation for neurogenic lower urinary tract dysfunction: systematic review and meta-analysis. Eur Urol 2010;58:865-74.

[30] Litwiller SE, Frohman EM, Zimmern PE. Multiple sclerosis and the urologist. J Urol 1999;161:743-57. 